\title{
Complex Erdheim-Chester disease associating rectal adenocarcinoma and breast cancer
}

\author{
Mihaela Guţư ${ }^{2 a}$, Mihai Danciu ${ }^{1,2 b}$, Mihai Guţu, ${ }^{* 1,2 a}$, Mihai Marinca ${ }^{1,3}$, Roxana \\ Livadariu $^{2 \mathrm{c}}$, Răzvan Tepordei ${ }^{1,2 \mathrm{~d}}$, Mioara $\mathrm{Nitu}^{2 \mathrm{e}}$, Tudor Cozma ${ }^{1,2 \mathrm{~d}}$, Manuela \\ Ursaru $^{1,2 e}$, Lucian Miron ${ }^{1,3}$, Cipriana Ştefănescu ${ }^{1,2 a}$
}

1"Gr. T. Popa" University of Medicine and Pharmacy lasi, Romania, ${ }^{2}$ University Emergency Hospital "Sf. Spiridon" lasi, Romania, ${ }^{a}$ Nuclear Medicine Department, ${ }^{b}$ Pathology Department, "General Surgery Department, ${ }^{\mathrm{d}}$ Orthopaedics and Traumatology Department, ${ }^{\mathrm{e}}$ Radiology Department,

${ }^{3}$ Regional Oncology Institute, lasi, Romania

\begin{abstract}
Erdheim-Chester Disease (ECD) is a rare form of non-Langerhans' cell histiocytosis (non-LCH). It is characterized by the excessive production of histiocytes. We present the case of a 63 year-old Caucasian woman with ECD, with a history of breast cancer (diagnosed in 2006) and rectal cancer (diagnosed in 2013). Most probably, the initial ECD manifestation occurred within the next 5 years after mastectomy in the soft tissues adjacent to the scar. Recently it became manifest in a different localization (meta-diaphysis of long bones), while the other possible sites like the lungs, central nervous system and pituitary gland show minimal involvement with no specific clinical manifestation yet. Shortly after the bone manifestations were detected, she developed an invasive rectal adenocarcinoma. The ECD diagnosis was based on bone scintigraphy, radiography and immunohistochemistry examination.
\end{abstract}

Keywords: Erdheim-Chester disease, breast cancer, rectal adenocarcinoma, scintigraphy, immunohistochemistry

\section{Introduction}

Histiocytic disorders are a group of diseases that occur when there is an overproduction of tissue macrophage cells known as histiocytes. The Erdheim-Chester Disease (ECD) (also known as polyostotic sclerosing histiocytosis) is a rare form of nonLangerhans cell histiocytosis (non-LCH) and until 2015 around 500 cases were reported [1].

Received: January 2016; Accepted after review: February 2016; Published: March 2016.

*Corresponding author: Mihai Guţu MD, PhD, "Gr. T. Popa" University of Medicine and Pharmacy, Nuclear Medicine Department, University Emergency Hospital "Sf. Spiridon", 700111, lasi, Romania.

Email: misugutu@yahoo.fr
Individuals affected by this disease are typically adults aged 50 to 70 years [2] but also 30 and 40 year-old cases were reported [3]. Males and females are almost equally affected, no clear gender-related differences in prevalence exist [2]. To our knowledge, no cases diagnosed with ECD that associate a malignancy (much less two) were yet reported; few cases were reported as ECD of the breast [4]. The ECD is characterized by the excessive production of histiocytes and so far it has no known etiology [5]. Sites of involvement include lung, bone, skin, retro-orbital tissue, central nervous system, pituitary gland, retroperitoneum and pericardium [1, 2]. The diagnosis is based on radiological and histological findings [2]; some authors suggest that bone scintigraphy could be the gold standard to assess bone involvement [6]. The 
radiological findings are diffuse bone sclerosis that occurs bilaterally and symmetrically in the meta-diaphysis regions of the long bones [2, 7]; bone scintigraphy notes increased tracer uptake particularly involving meta-diaphysis of the femurs and tibias [8]. The histological changes include tissue infiltration with lipoid granulomas composed of foamy CD68positive but CD1a-negative macrophages, in contrast to Langerhans cells. These macrophages are also usually negative for $\mathrm{S}$ 100 protein but may occasionally be weakly positive $[9,10]$.

We report a patient with a typical pattern of bone-generalized ECD, later proven to have mammary involvement, which was associated with breast and rectal cancer. The diagnosis was based on bone scintigraphy, clinical identification of a breast lump and bone biopsies (histological/ immunohistochemical findings). Mammary involvement by ECD is an extremely rare condition, which should be differentiated from benign or malignant tumors. Distinctive histological features plus clinical and scintigraphic information are needed to achieve a correct diagnosis [2].

\section{Case report}

A 63 year-old Caucasian woman with a complex medical history, had addressed to Nuclear Medicine Department for investigation of a recently onset knee pain.

She was diagnosed at age 55 (December 2006) with a nodule in the upper outer quadrant of her left breast. Quadrantectomy with axillary dissection was performed, and pathology showed invasive carcinoma of no specific type (IC-NST) with ductal carcinoma in situ (DCIS); nine of a total 25 excised lymph nodes were positive for tumor cells (TNM stage IIIA).

Immunohistochemistry revealed intensely positive estrogen (90\% of tumor cells) and progesterone receptors $(75 \%)$ and negative (1+) Her-2/neu status. The patient was administered 6 cycles of adjuvant chemotherapy (standard FE90C protocol) and radiation therapy (60 $\mathrm{Co}$, a total dose of $60 \mathrm{~Gy}$ in 30 fractions on the tumor bed, left breast and axilla), then continued on hormone therapy (anastrozole 1mg/day). In February 2008, a breast ultrasound showed a 19-mm mass underneath the surgical scar. Pathology exam of the lumpectomy specimen showed a IC-NST with foci of DCIS and mucinous carcinoma. After 3 cycles of paclitaxel monochemotherapy, a 40-mm cyst-like mass of DCIS was removed through left Madden modified radical mastectomy in April 2008. She was then administered another 4 cycles of chemotherapy (docetaxel plus gemcitabine) and continued on anastrozole for a total of 5 years. During follow-up (January 2012), a clinical examination identified two $30 / 20-\mathrm{mm}$ tumors on the thoracic wall and basis of the right axilla, respectively, which were concomitantly excised. Pathology report diagnosed a multicentric benign fibrous histiocytoma (BFH) - a subtype of $\mathrm{LCH}$. Reexcision was needed in March 2012 and again in January 2013, with the same diagnosis of BFH. Starting July 2013, she complained of abdominal pain, weight loss and fatigue, and also bilateral knee pain. A digital rectal examination (October 2013) identified a tumor at $30-40 \mathrm{~mm}$ from the anal verge, clinically staged as $T_{3} N_{2} M_{0}$ by chest and abdominal CTscans; biopsy was diagnostic for well differentiated rectal adenocarcinoma.

A bone biopsy was performed from the distal femoral lesions. All tissue specimens were routinely processed using formalin fixation and paraffin embedding. Additionally, after fixation, bone tissue specimens taken from inferior metaphysis of the right femur were decalcified. Five-micron tissue sections were examined under routine - hematoxylineosine (HE) and trichromic - staining. Immunohistochemistry used monoclonal antibodies anti-CD68 (clone PG-M1, Dako), anti-CD1a (clone 010, Dako), anti-Cytokeratin (clone AE1/AE3, Dako) and polyclonal antibody anti-S100 protein (Dako). To exclude a breast cancer metastasis to the bone, estrogen receptor (clone 1D5, Dako) and progesterone receptor (clone PgR 636, Dako) staining was also used. Tissue pretreatment included heat-induced epitope retrieval in citrate buffer solution, and detection was done with an EnVision kit. 


\section{Discussion}

The almost symmetrically distributed bone pain (especially in the knees) that could have suggested the ECD diagnosis - as described in other cases [7] - had appeared 2 years later. In this context the radiology (knee X-ray) revealed a symmetrical femoral metadiaphysis fibrosis (also common in ECD) $[2,6]$ (Figure 1).

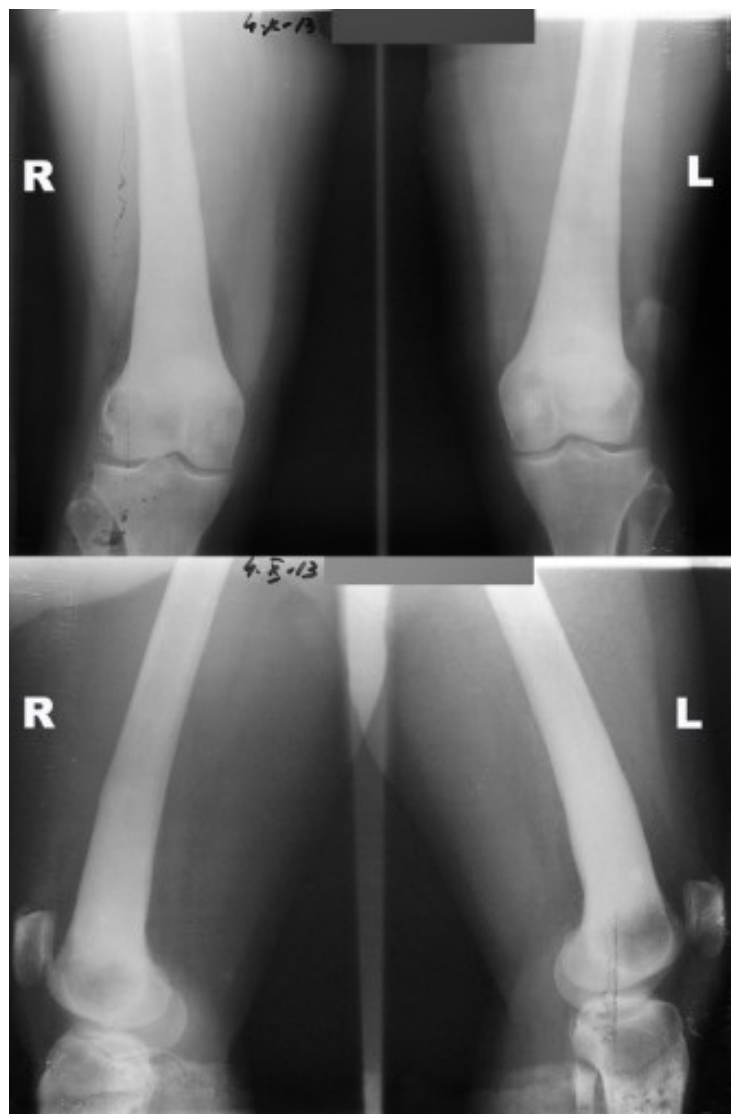

Fig. 1. Knee X-ray showing bilateral and symmetrical bone condensation along the femoral metadiaphysis

The long bones fibrotic reaction was showed by the high uptake on bone scintigraphy for distal femoral and proximal tibia meta-diaphysis, sparing the epiphysis. The bilateral tibiae uptake was very inhomogeneous, lytic areas on the right anterior tuberosity and left diaphysis suggesting a possible association with $\mathrm{LCH}$ or Rosai-Dorfman Disease (RDD) [2, 8]. Other pathological uptake areas were the left clavicle, right maxilla, bilateral radial and ulnar diaphysis and metaphysis. Moderate uptake was also seen on the left proximal and right distal humeral metaphysis and the left greater trochanter (Figure 2). Brain MRI showed thickening in the upper two thirds of pituitary stem, adjacent to the posterior optical chiasm. Since pathogenesis, natural history and lesions are very similar for $\mathrm{LCH}$ and non-LCH, both showing $\mathrm{LCH}$ multisystem involvement and a possible abnormality in the common CD34 progenitor cell [11], a bone biopsy and microscopic re-examination of soft tissue samples biopsied from the left axillary region and anterior thoracic region in 2012, with subsequent tissue processing and immunohistochemistry were done for differential diagnosis of LCH, EDC, RDD and bone metastasis. The breast tissue examination identified a fibro-histiocytic proliferation with numerous foamy cells, some multinucleated and Touton giant cells, surrounded by lymphocytes. Rare mitotic figures were observed; architecture was focally storiform (similar to that of dermatofibrosarcoma). In the periphery, the tumor infiltrated the skeletal muscle and adipose tissue. Breast ECD is otherwise a rarely described localization [4, 12], ours being only the seventh such case report to our knowledge [13]. Microscopic examination of the bone biopsy revealed the replacement of the fatty marrow by fibrosis (Figure $3 \mathrm{E}$ ) and foamy cells: nests of large cells, with small central nuclei and finely granular, pale eosinophilic cytoplasm. The ECD is characterized by xanthomatous or xanthogranulomatous infiltration of tissues by foamy histiocytes, "lipid-laden" macrophages, or histiocytes, surrounded by fibrosis [1] (Figure 3A). No multinucleated or Touton giant cells were identified in our sections.

While ECD and RDD could be differentiated pathologically (lack of emperipolesis) or clinically (no massive lymphadenopathy) [14], immunohistochemistry performed on bone marrow (or soft) tissue is essential in distinguishing Langerhans cells from non-Langerhans histiocytes. Immunohistochemical reassessment of the breast lesion revealed the following patterns: CD68 (+), CD1a (-), S100 (-), and CD68 (+), CD1a (-), S100 (+/-), respectively. The initial diagnosis (cellular $\mathrm{BFH}$ ) was reconsidered as 
non-LCH. The similar immunohistochemical profile in the bone - CD68 (+), CD1a (-) [2] and S100 (slightly positive) [10] - also supported the non-LCH type of bone proliferating histiocytes. The mild and focal positivity of $\mathrm{S} 100$ in histiocytes and the absence of emperipolesis in those histiocytes expressing $\mathrm{S} 100$ protein rule out the diagnosis of RDD. The negative staining for antibodies against cytokeratin (AE1/AE3 cocktail), estrogen (ER) and progesterone receptors (PgR) excluded a breast cancer metastasis. CD1a was negative in foamy histiocytes (Figure 3B), S100 was focally positive in foamy histiocytes and adipocytes (Figure $3 \mathrm{C}$ ), and CD68 was positive in all foamy histiocytes (Figure 3D). Histological aspects and immunohistochemical profile of all lesions were highly consistent with the diagnosis of ECD.
It is important to remember that between first surgery and the soft tissue recurrence, our patient underwent chemo-, radio- and endocrine therapy. In this context, one might wonder whether the primary breast tumor could have also contained occult foci of ECD in the comedo type areas. As a result of radiotherapy [12] and/or the systemic treatments [15] these might have slowly evolved before becoming clinically evident. Radiotherapy has proven high rates of local control and symptomatic improvement in $\mathrm{LCH}$, including some ECD patients [16, 17]. Chemotherapeutic agents have not been reliably evaluated as possible treatment for either ECD or LCH. Hormonal therapy for breast cancer is interfering with bone metabolism and might slow down the evolution of ECD [18].

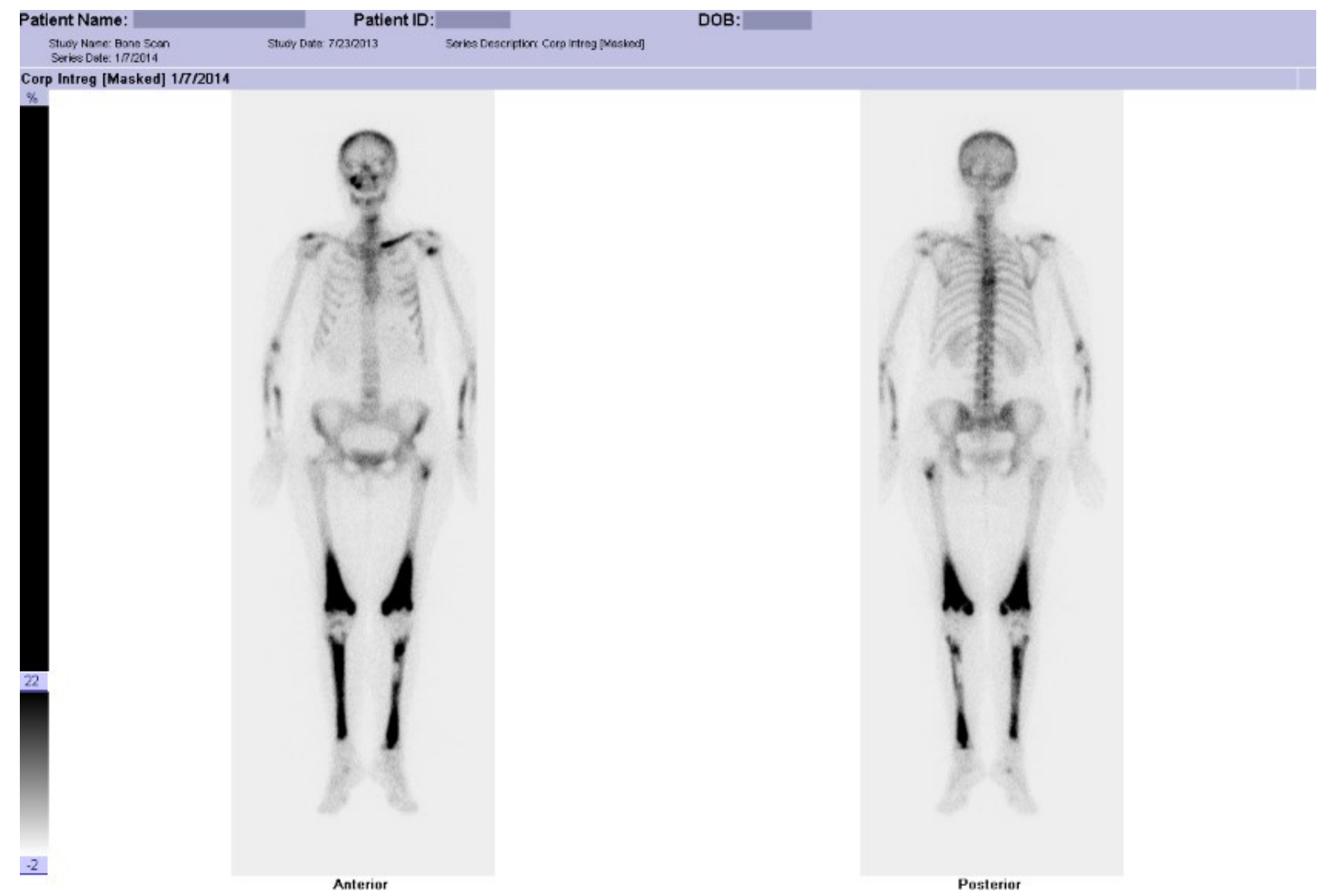

Fig. 2. Whole body bone scintigraphy. High uptake on long bones: distal femoral diaphysis, tibiae diaphysis and metaphysis spearing the epiphysis. Other pathological uptakes can be seen on left clavicle, right maxilla, bilateral radial and ulnar diaphysis and metaphysis 


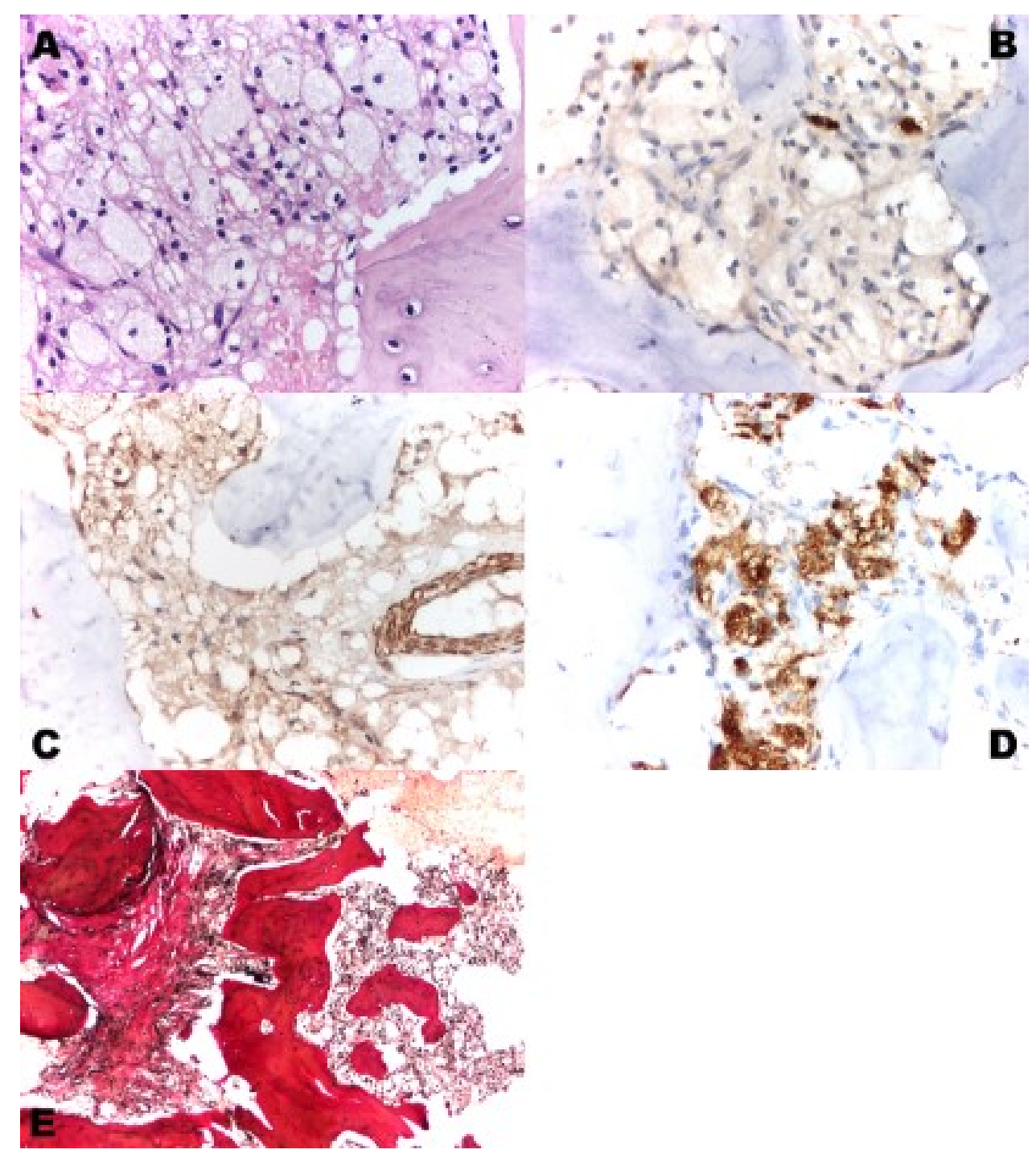

Fig. 3. Histological aspects and immunohistochemical profile of the bone marrow. A. Foamy histiocytes, "lipidladen" macrophages (HE, x200). B. CD1a (-) in foamy histiocytes (IHC, Ab Anti-CD1a, x200). C. S100 (+) (focally) in foamy histiocytes and adipocytes (IHC, Ab Anti-S100, x200). D. CD68 (+) in all foamy histiocytes (IHC, Ab Anti-CD68, x200). E. Replacement of the fatty marrow by fibrosis and foamy cells (van Gieson trichromic, $\mathrm{x} 40)$.

The initial manifestation of the ECD in this case was a focal breast soft tissue lesion. Another interesting fact is that, although multisystemic infiltration of critical organ exists, it is not yet clinically manifest, despite the high osseous uptake of the Tc99m-hydroxybisphosphonate and the time passed from the first histological manifestation. While we consider the rectal adenocarcinoma to be a purely coincidental comorbidity, to our knowledge, this is the first published case of ECD associated with breast and rectal cancer. The patient is now following neoadjuvant chemo-radiotherapy. Decisions on further treatment will be made after radical surgery for rectal cancer. Not the least important of these decisions is the one on interferon therapy (the currently accepted treatment in ECD and other types of histiocytosis). Whether this should be initiated right away (should the clinical condition permit), deferred for a 1- or 2-year interval or forgone altogether (given the potential negative impact of immune system modulation on residual cancer cells) remains a matter of discussion. 


\section{Conclusion}

ECD is a rare disease and its clinical manifestation could be very unspecific in oncological context. Bone scintigraphy (more sensitive than radiography) could be the starting point for ECD diagnosis, and should be followed by pathology examination and immunohistochemical tests. Specific features

\section{References}

1. Haroche J, Arnaud L, Cohen-Aubart F, et al. Erdheim-chester disease. Curr Rheumatol Rep 2014; 16:412.

2. Mazor RD, Manevich-Mazor M, Shoenfeld $Y$. Erdheim-Chester Disease: a comprehensive review of the literature. Orphanet $J$ Rare Dis 2013; 8:137.

3. Al-Quran S, Reith J, Bradley J, Rimsza L. Erdheim-Chester disease: case report, PCRbased analysis of clonality, and review of literature. Mod Pathol 2002; 15:666-672.

4. Barnes PJ, Foyle A, Hache KA, et al. ErdheimChester disease of the breast: a case report and review of the literature. Breast $J$ 2005; 11:462-467.

5. Chetritt J, Paradis V, Dargere D, et al. ChesterErdheim disease: a neoplastic disorder. Hum Pathol 1999; 30:1093-1096.

6. Aouba A, Bienvenu B, Launay D, Hermine O. Role of iconographic examinations in the treatment algorithm in Erdheim-Chester disease. J Clin Oncol 2011; 29:4466-4467; author reply 4468-4469.

7. Charest M, Haider EA, Rush C. An unusual cause of knee pain. Br J Radiol 2007; 80:227229.

8. Ceulemans $G$, Keyaerts M, Verbruggen L, et al. Erdheim-Chester disease detected with 99mTc MDP bone SPECT/CT. JBR-BTR 2012; 95:245-248.

9. Shimada $S$, Ono $K$, Hashizume $Y$, et al. Intracranial lesion of Erdheim-Chester disease. Hum Pathol 2007; 38:950-951.

10. Ono K, Oshiro M, Uemura K, et al. ErdheimChester disease: a case report with immunohistochemical and biochemical examination. Hum Pathol 1996; 27:91-95. of this particular patient are: breast cancer history, ECD emerging in the breast soft tissue adjacent to postsurgical scars and association of a rectal adenocarcinoma. This case could make a point for routine ECD-specific immunohistochemical tests, at least in selected cases of breast cancer with local recurrence alone.

11. Pineles SL, Liu GT, Acebes $X$, et al. Presence of Erdheim-Chester disease and Langerhans cell histiocytosis in the same patient: a report of 2 cases. J Neuroophthalmol 2011; 31:217-223.

12. Andrade VP, Nemer CC, Prezotti AN, Goulart WS. Erdheim-Chester disease of the breast associated with Langerhans-cell histiocytosis of the hard palate. Virchows Arch 2004; 445:405409.

13. Furuta $\mathrm{T}$, Kiryu S, Yamada $\mathrm{H}$, et al. ErdheimChester disease with an 18Ffluorodeoxyglucose-avid breast mass and BRAF V600E mutation. Jpn J Radiol 2014; 32:282-287.

14. Sandoval-Sus JD, Sandoval-Leon AC, Chapman JR, et al. Rosai-dorfman disease of the central nervous system: report of 6 cases and review of the literature. Medicine (Baltimore) 2014; 93:165-175.

15. Jendro $M C$, Zeidler $H$, Rosenthal $H$, et al. Improvement of Erdheim-Chester disease in two patients by sequential treatment with vinblastine and mycophenolate mofetil. Clin Rheumatol 2004; 23:52-56.

16. Kotecha $R$, Venkatramani $R$, Jubran $R F$, et al. Clinical Outcomes of Radiation Therapy in the Management of Langerhans Cell Histiocytosis. Am J Clin Oncol 2014; 37(6):592-596.

17. Matsui K, Nagata $Y$, Hiraoka M. Radiotherapy for Erdheim-Chester disease. Int J Clin Oncol 2007; 12:238-241.

18. Servitja $S$, Nogues $X$, Prieto-Alhambra D, et al. Bone health in a prospective cohort of postmenopausal women receiving aromatase inhibitors for early breast cancer. Breast 2012; 21:95-101. 